Tamaño de la concha de Notoacmea biradiata (Archaeogastropoda: Acmaeidae) como respuesta a la densidad de gasterópodos y altura intermareal en la costa pacífica de Colombia

\title{
Shell-size variation of Notoacmea biradiata (Archaeogastropoda: Acmaeidae) as a response to gastropod density and height in shore in the Colombian Pacific coast
}

\author{
Alan Giraldo ${ }^{1,3}$ \\ Carolina Gómez ${ }^{2}$ \\ Efraín Rodríguez ${ }^{3}$ \\ ${ }^{1}$ Universidad del Valle \\ Departamento de Biología \\ Sección de Biología Marina. A.A. 25360 \\ Cali, Colombia \\ ${ }^{2}$ Universidad Autónoma de Occidente \\ Programa Admón. del Medio Ambiente y de los Recursos Naturales \\ Campus Valle del Lilí, Calle 25 \#115-85 \\ Cali, Colombia \\ ${ }^{3}$ Universidad de Concepción \\ Departamento de Oceanografía \\ Casilla $160 \mathrm{C}$ \\ Concepción, Chile \\ *E-mail: agiraldo@biologos.univalle.edu.co
}

Recibido en julio de 2001; aceptado en abril de 2002

\section{Resumen}

Notoacmea biradiata (Reeve, 1855) es un gasterópodo patelado abundante en las playas rocosas intermareales del Pacífico colombiano. En este estudio se presentan los resultados de un experimento factorial sobre la variación en el tamaño de la concha de $N$. biradiata como respuesta a dos factores independientes: la densidad de gasterópodos intermareales y la altura intermareal. Para el estudio, se seleccionaron cuatro playas rocosas intermareales en el Pacífico colombiano, dos en la Isla Gorgona y dos en la Ensenada de Utría. Se determinó la densidad de gasterópodos intermareales en cada sitio utilizando la técnica del transectocuadrante. Se encontraron diferencias significativas de densidad entre los sitios de estudio: baja densidad en las playas rocosas de la Ensenada de Utría y alta densidad en las playas rocosas de la Isla Gorgona $(P<0.009)$. Se realizó un muestreo factorial para evaluar el efecto de la densidad de gasterópodos y la altura intermareal sobre la longitud, altura y grosor de la concha. Se utilizó un análisis de varianza de dos vías y un análisis de regresión lineal para establecer las diferencias en el tamaño de la concha debido a la densidad o a la altura intermareal. Los resultados obtenidos sugieren que la densidad de gasterópodos intermareales determina las variaciones de la longitud de la abertura $(P<0.001)$ y altura de la concha $(P<0.05)$, encontrando conchas grandes y altas en zonas de baja densidad, mientras que la altura intermareal no fue fuente de varianza para el tamaño de la concha (ANOVA, $P>0.30$ ).

Palabras claves: Notoacmea, patelas, playa rocosa intermareal, Océano Pacífico, Colombia.

\begin{abstract}
Notoacmea biradiata (Reeve, 1855) is an abundant intertidal limpet on the rocky shore of the Colombian Pacific coast. This paper reports a field factorial experiment on shell-size variation of $N$. biradiata as a response to two independent factors: intertidal gastropod density and shore height above sea level. Four rocky shores were selected on the Colombian Pacific coast, two in Gorgona Island and two in Utría Cove. The intertidal gastropods density was determined in each rocky shore using the transect-quadrant technique. Significant differences were observed in the gastropod densities between study sites: low density in the rocky shores of Utría Cove and high density in the rocky shores of Gorgona Island $(P<0.009)$. A factorial sampling was conducted to determine the effect of the density and height on the shore over shell-length, shell-height and shell-thickness. A twoway ANOVA and a least-squared regression was used to determine whether the shell-sizes differ between varying densities and
\end{abstract}


shore heights. Intertidal gastropod density was found to be the dominant factor in determining basal shell length (ANOVA, $P<0.001$ ) and shell height (ANOVA $P<0.05$ ) changes. Thus, at low population densities the shells are larger and taller. The height on the shore did not influence the shell-size of $N$. biradiata (ANOVA, $P>0.30$ ).

Keywords: Notoacmea, limpet, intertidal rocky shore, Pacific Ocean, Colombia.

\section{Introducción}

Las playas rocosas intermareales presentan un gradiente ambiental marcado, en distancias tan cortas, que las poblaciones de moluscos pueden interactuar simultáneamente con diferentes componentes físicos y biológicos del ambiente. En estas zonas, el régimen mareal determina los gradientes de temperatura, desecación y tiempo de inmersión, los cuales afectan a los organismos que allí habitan (Black, 1979; Levinton, 1984). Por lo tanto, factores biológicos como densidad y predación, y factores físicos como intensidad del oleaje, desecación y altura intermareal pueden ser considerados como factores modificadores de las comunidades que habitan la zona intermareal en un amplio rango de las escalas de organización ecológica (Lewis, 1964).

La diversidad morfológica y las estrategias funcionales entre los organismos intermareales son una fuente importante de información para realizar estudios comparativos (Ortega, 1985). Además, el ambiente físico es lo suficientemente severo para permitir evaluar la relación entre una estructura biológica y su función en un ambiente específico (Denny, 1994). Factores como la predación (Heller, 1976; Garrity y Levings, 1983), desecación (Spencer, 1969; Branch, 1975a; Lowell, 1984), ritmos de actividad (Branch y Cherry, 1985), altura intermareal (Vermeij, 1972; Butler 1979; Hobday, 1995), comportamiento migratorio (Hobday, 1995), y la intensidad del oleaje (Brown y Quinn, 1988; Denny, 1994; De Wolf et al., 1999; Giraldo y Gómez, 1999) tienen influencia sobre la variación en el tamaño de la concha. Además, la cantidad de alimento y la disponibilidad de espacio son importantes para el crecimiento de las patelas. Sin embargo, ambos factores están estrechamente relacionados con la densidad de gasterópodos intermareales (Nybakken, 1997). Por lo tanto, la densidad debe ser un factor importante en la variación intraespecífica del tamaño de la concha.

La desecación es otro de los factores importantes en la variación del tamaño de la concha. Este factor está asociado directamente con la altura intermareal. Los gasterópodos que habitan la zona intermareal alta están expuestos durante más tiempo al aire y a las altas temperaturas con relación a los congéneres que habitan la zona intermareal baja. En las patelas se ha observado una relación directa entre la mortalidad y la desecación (Frank, 1965). Sin embargo, una amplia tolerancia a la desecación podría ser una ventaja para las patelas, permitiéndoles alcanzar recursos alimentarios no explotados y mantenerse por más tiempo en las zonas de reproducción (Frank, 1965; Vermeij, 1972; Wolcott, 1973; Blackford y Cook, 1978; Underwood, 1979; Bertness et al., 1981; Garrity y Levings, 1983).

\section{Introduction}

The intertidal rocky shore creates a steep environmental gradient over distances so short that gastropods populations can interact simultaneously with different physical and biological components of the environment. Thus, the tidal regime determines temperature, desiccation and submersion time gradients, those which influence the organisms that inhabit intertidal shores (Black, 1979; Levinton, 1984). Therefore, biological factors like density or predation and physical factors like wave intensity, desiccation or shore height, can be considered as modifying factors which act on intertidal zone inhabiting communities over a wide range of ecological organizational scale (Lewis, 1964).

Morphological diversity and functional strategies among intertidal organisms provide a natural basis for comparative studies (Ortega, 1985). Furthermore, the physical environment is so severe that it would be useful to evaluate the match between a biological structure and its function, in a specific environment (Denny, 1994). Predation (Heller, 1976; Garrity and Levings, 1983), desiccation (Spencer, 1969; Branch, 1975a; Lowell, 1984), activity rhythms (Branch and Cherry, 1985), shore height (Vermeij, 1972; Butler, 1979; Hobday, 1995), migratory behavior (Hobday, 1995), and wave intensity (Brown and Quinn, 1988; Denny, 1994; De Wolf et al., 1999; Giraldo and Gómez, 1999) are factors that have been reported to influence the gastropod shell-sizes. On the other hand, food quantity and space availability are important for limpet growth. However, both factors are affected by the density of intertidal gastropods (Nybakken, 1997). Then, the density should play an important role on intraspecific shell-size variability.

Desiccation is another main factor on shell-size variation. This factor is directly associated with the height on the shore. Gastropods inhabiting the upper intertidal zone are exposed for longer periods to air and higher temperatures than those in the lower intertidal zone. Mortality due to desiccation has been slightly observed in limpets (Frank, 1965). However, a wide tolerance to desiccation would be an adaptative advantage for the limpets, allowing them to reach unexploited food resources and to remain in breeding zones (Frank, 1965; Vermeij, 1972; Wolcott, 1973; Blackford and Cook, 1978; Underwood, 1979; Bertness et al., 1981; Garrity and Levings, 1983).

The limpet Notoacmea biradiata (Reeve, 1855) is an herbivorous and abundant intertidal gastropod that is found in the Pacific coast from Mexico to Ecuador. It has a low shield-like and un-ribbed shell. The shell is brownish with two distal broad white lines radiating from the apex (Keen, 1971). Considering that intertidal limpets are a good model to study the effects of external factor on the gastropod shell-size variability 
Notoacmea biradiata (Reeve, 1855) es un gasterópodo patelado herbívoro que se encuentra en la costa pacífica americana desde México hasta Ecuador. Su concha es pequeña, café oscura, no presenta marcas o costillas externas y posee dos líneas anchas blancas en la parte posterior que irradian desde el ápice (Keen, 1971). Considerando que los gasterópodos patelados intermareales son un buen modelo para estudiar los efectos de factores externos sobre la variabilidad de la concha en ambientes intermareales (Ortega, 1985), en esta investigación se describen los patrones de densidad de gasterópodos intermareales en dos localidades de la costa pacífica colombiana y se evalua el efecto de la densidad de gasterópodos intermareales y la altura intermareal sobre el tamaño de la concha en $N$. biradiata. Las preguntas que se abordaron en este estudio son: (a) ¿Es la densidad de los gasterópodos intermareales significativamente diferente entre las áreas de estudio?, (b) ¿Afecta la densidad de los gasterópodos intermareales el tamaño de la concha de $N$. biradiata?, y (c) ¿Es la altura intermareal un factor determinante en la variación del tamaño de la concha en $N$. biradiata?

\section{Materiales y métodos}

\section{Área de estudio}

La costa pacífica colombiana $\left(77^{\circ} 53^{\prime} \mathrm{W}, 7^{\circ} 13^{\prime} \mathrm{N}-78^{\circ} 48^{\prime} \mathrm{W}\right.$, $\left.1^{\circ} 26^{\prime} \mathrm{N}\right)$ tiene una extensión de $1542 \mathrm{~km}$. La zona norte se caracteriza por depósitos terciarios (e.g. magmatitas) y la zona sur por depósitos cuaternarios (e.g. sedimentitas) (Anónimo, 1983). Estas características geológicas definen la morfología costera: la zona norte presenta acantilados con costas rocosas amplias, mientras que la zona sur es plana con playas arenofangosas (Rodríguez-Rubio, 1997). El ambiente físico esta determinado por la co-ocurrencia de la zona de convergencia intertropical (ITCZ) y la zona ecuatorial de baja presión (ELPT). En resumen, desde diciembre hasta mayo los vientos alisios provienen del noreste mientras que de junio a noviembre provienen del sureste. La precipitación media es de $5691 \pm$ $1857 \mathrm{~mm}$ año ${ }^{-1}$, la escorrentía es de $4151 \pm 1751 \mathrm{~mm}$ año ${ }^{-1}$ (promedio anual de estaciones climáticas a lo largo de la costa pacífica colombiana, $n=11$ ), la temperatura promedio del aire es de $26 \pm 1^{\circ} \mathrm{C}$ y la humedad relativa alcanza $85 \%$ (Forsbergh, 1969; Pabón et al., 1998; Tchantsev y Cabrera, 1999). La temperatura del agua en la zona costera es $28.86 \pm 1.45^{\circ} \mathrm{C}(n=13) \mathrm{y}$ la salinidad es de $21.98 \pm 8.25(n=24)$ (Forsbergh, 1969), sin embargo, estas condiciones se alteran drásticamente cuando ocurre El Niño (Tchantsev y Cabrera 1999).

Este estudio se realizó en dos localidades de la costa pacífica colombiana (fig. 1). En cada localidad se seleccionaron dos áreas de estudio: Playa Blanca (PR-1) y la Camaronera

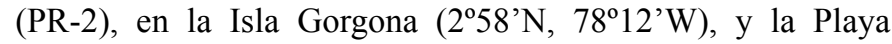
Salomón (PR-3) y Cocalito (PR-4), en la Ensenada de Utría $\left(6^{\circ} 02^{\prime} \mathrm{N}, 7^{\circ} 20^{\prime} \mathrm{W}\right)$. El sustrato estuvo conformado por rocas sedimentarias y el periodo mareal fue semidiurno (rango extremo mareal: $3.50 \mathrm{~m}$ ). Se refirió el nivel intermareal con
(Ortega, 1985), in this paper we describe the pattern of intertidal gastropod density in two localities of the Pacific coast of Colombia, and we studied the density and shore height effects on $N$. biradiata shell-size variability. The specific questions covered in this study were: (a) Is the intertidal gastropod density significantly different between study sites?, (b) How much is $N$. biradiata shell-size affected by intertidal gastropod density?, and (c) Is the intertidal shore height an important factor for the variation of the shell size of $N$. biradiata?

\section{Materials and methods}

\section{Study site}

The Pacific coast of Colombia ( $77^{\circ} 53^{\prime} \mathrm{W}, 7^{\circ} 13^{\prime} \mathrm{N}-78^{\circ} 48^{\prime} \mathrm{W}$, $1^{\circ} 26^{\prime} \mathrm{N}$ ) extends along $1542 \mathrm{~km}$. The northern zone is characterized by tertiary deposits (e.g. magmatites), while the southern zone is by quaternary deposits (e.g. sedimentites) (Anonymous, 1983). These geologic features define the coastal morphology: northern zones are steep with vast rocky shores, while the southern zone is flat with vast sand-muddy shores (Rodríguez-Rubio, 1997). The co-occurrence of the Inter-Tropical Convergence Zone (ITCZ) and the Equatorial Low Pressure Trough (ELPT) determine the physical environment. Synthesizing, trade winds come from the Northeast between December to May while the rest of the year they come from the Southeast. The mean rainfall is about $5691 \pm 1857 \mathrm{~mm}_{\text {year }}{ }^{-1}$ and runoff is about $4151 \pm 1751 \mathrm{~mm}^{\text {year }}{ }^{-1}$ (annual mean from the weather stations along the Pacific coast of Colombia, $n=$ 11); the mean air temperature is $26 \pm 1^{\circ} \mathrm{C}$ and the relative humidity reaches 85\% (Forsbergh, 1969; Pabón et al., 1998; Tchantsev and Cabrera, 1999). The mean near shore SST is about $26.86 \pm 1.45^{\circ} \mathrm{C}(n=13)$ and salinity about $21.98 \pm 8.25$ ( $n=24)$ (Forsbergh, 1969); however, this conditions widely change when the ENSO occurs (Tchantsev and Cabrera, 1999).

The study was carried out in two localities on the Pacific coast of Colombia (fig 1). In each locality, two study sites were selected: Playa Blanca (PR-1) and La Camaronera (PR-2) in Gorgona Island $\left(2^{\circ} 58^{\prime} \mathrm{N}, 78^{\circ} 12^{\prime} \mathrm{W}\right)$ and Playa Salomón (PR-3) and Cocalito (PR-4) in Utría Cove $\left(6^{\circ} 02^{\prime} \mathrm{N}, 77^{\circ} 20^{\prime} \mathrm{W}\right)$. In the study sites the substratum was sedimentary rocks and the tide period was semidiurnal (extreme tidal range: $3.50 \mathrm{~m}$ ). The shore height was referred in relation to mean low water (MLW) as: low (0.00-1.70 $\mathrm{m}$ above MLW), mid (1.70-2.50 $\mathrm{m}$ above MLW) and high (2.50-3.50 $\mathrm{m}$ above MLW).

\section{Methods}

The density of common intertidal gastropods was established in each shore height of the studied areas, using a random quadrant $\left(0.96 \mathrm{~m}^{2}\right)$ throughout three transects across the shore. A total of 18 quadrants from each study site were evaluated. A two-way ANOVA test was used to determine whether the mean density differed at each shore height or between the study sites 
relación a la media de agua más baja de marea (MLW) como: zona intermareal baja (0.00-1.70 m MLW), zona intermareal media (1.70-2.50 m MLW) y zona intermareal alta (2.50-3.50 m MLW).

\section{Método}

Se determinó la densidad de gasterópodos en los tres niveles intermareales de cada área de estudio, utilizando un cuadrante de $0.96 \mathrm{~m}^{2}$ de manera aleatoria a lo largo de tres transectos perpendiculares a la costa; para un total de 18 cuadrantes en cada área de estudio. Se realizó un análisis de varianza de dos vías (ANOVA) para determinar si las densidades medias diferían entre alturas intermareales o entre localidades, con el propósito de establecer dos niveles contrastantes de densidad.

Los individuos de $N$. biradiata fueron removidos manualmente durante la marea baja utilizando una lámina de acero inoxidable, registrando la altura intermareal en la que se encontraron. Se midió la longitud de la base, la altura desde la base hasta el ápice y el grosor de la concha utilizando un calibrador vernier $(0.05 \mathrm{~mm})$. Para determinar si el valor promedio de las características morfológicas de la concha diferían con relación a la densidad o altura intermareal se realizó un análisis de varianza de dos vías. Se examinó la homocedasticidad de varianza utilizando la prueba estadística de Bartlett y se realizó una transformación logarítmica cuando fue necesario. Si esta condición no se cumplía, se realizó una prueba de comparación de medias no paramétrica o prueba de Kruskall-Wallis (Zar, 1999). Se estimó la ecuación de regresión entre la longitud de abertura-altura de la concha (SHBL) y longitud de aberturagrosor de la concha (STBL) (Edbling et al., 1962; Giraldo y Gómez, 1999) bajo cada condición de densidad, y se compararon las asociaciones mediante un análisis de covarianza (ANCOVA).

\section{Resultados}

Las densidades medias ( \pm una desviación estándar) para cada una de las áreas de estudio se presentan en la figura 2. El número de gasterópodos fue mayor en las playas rocosas de Isla Gorgona que en las playas rocosas de la Ensenada de Utría (ANOVA: $F_{3,66}=7.41, P<0.001$; test de Tukey: $P<0.009$ ), definiendo dos niveles contraste de densidad. Se encontraron diferencias significativas de densidad entre las zonas intermareales, siendo más abundantes los gasterópodos en la zona intermareal alta (ANOVA: $F_{2,66}=8.94, P<0.001$; test de Tukey: $P<0.001)$. Sin embargo, no se detectó diferencia significativa de densidad entre la zona intermareal media y baja en las áreas de estudio (ANOVA: $F_{2,66}=2.45, P>0.140$ ). Los gasterópodos intermareales observados durante los muestreos fueron principalmente de especies herbívoras: patelas en la zona intermareal baja y media, y litorinas en la zona intermareal alta. Aunque, se detectaron gasterópodos predadores con

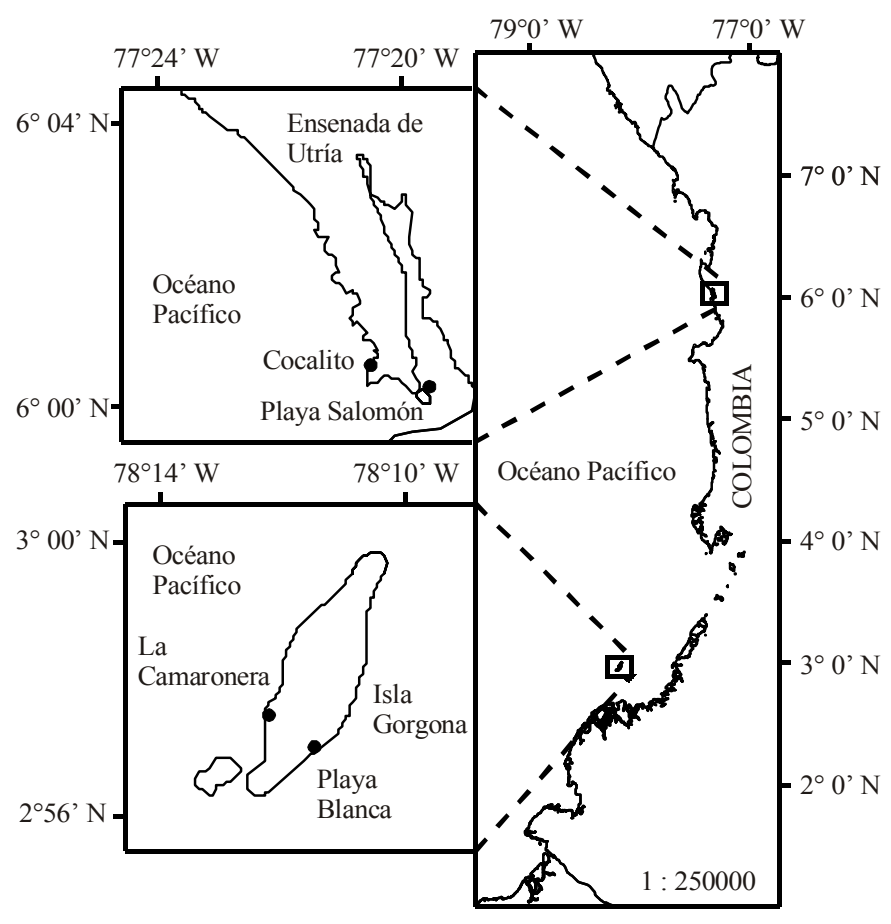

Figura 1. Ubicación geográfica del área de estudio en la costa pacífica colombiana. También se indica la ubicación de las playas rocosas seleccionadas en cada localidad.

Figure 1. Geographical location of the study area in the Colombian Pacific coast. The figure also shows the location of the rocky shore at each locality.

too, in order to define two contrasting density levels.

Notoacmea biradiata limpets were manually removed during low tides using a stainless steel sheet, and the intertidal shore height was registered. The base shell-length, shell-height from base to apex and shell-thickness were measured with a vernier caliper $(0.05 \mathrm{~mm})$. A two-way ANOVA test was used to determine if the average characteristics of every shell differed at each density or shore height level. The homoscedasticity was examined with the Bartlett's test and the logarithmic transformation was performed when necessary (Zar, 1999). If this condition was not fulfilled, a non-parametric mean comparison, or Kruskal-Wallis test, was used. The least-squared regression between shell-height $v s$ basal shell-length (SHBL) and shell-thickness $v s$ basal shell-length (STBL) at each density level was calculated (Edbling et al., 1962; Giraldo and Gómez, 1999). An ANCOVA test was used to compare the resulting equations.

\section{Results}

The mean density distribution ( \pm one standard deviation) in each study site is summarized in figure 2 . The intertidal gastropod density was higher in the rocky shores of Gorgona Island than in those of Utría Cove $\left(F_{3,66}=7.41, P<0.001\right.$; Tukey-test: $P<0.009$ ), defining two contrasting density levels. Significant variation in gastropod density was detected between shore 


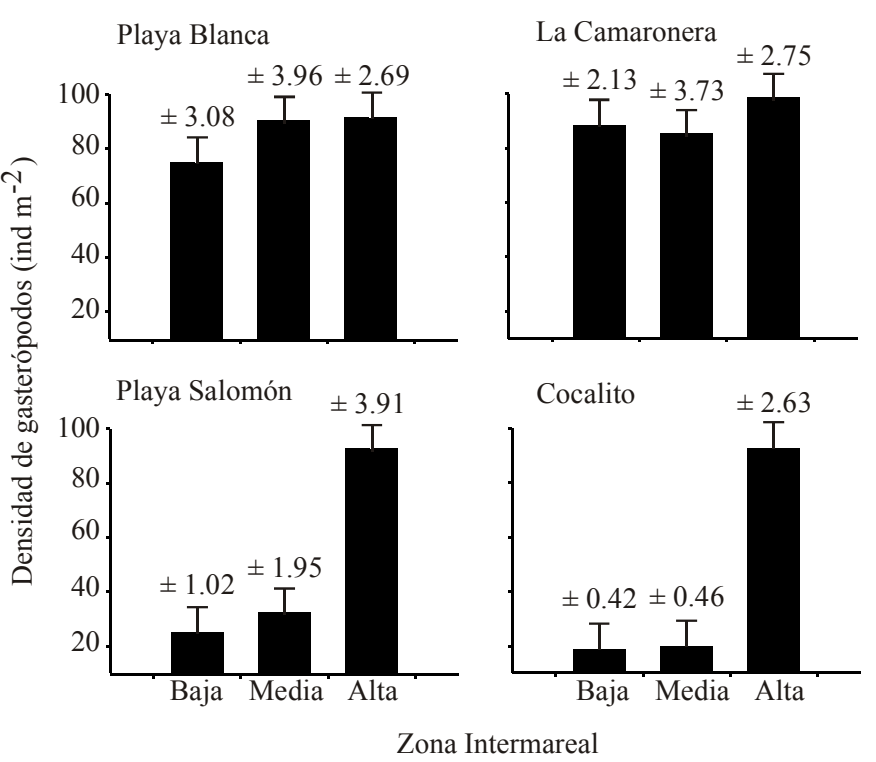

Figura 2. Densidades medias de gasterópodos para cada zona intermareal en la Isla Gorgona (PR-1, PR-2) y la Ensenada de Utría (PR-3, PR-4). La barra vertical indica \pm una desviación estándar.

Figure 2. Gastropod density means for each intertidal shore height at Gorgona Island (PR-1, PR-2) and Utría Cove (PR-3, PR-4). The horizontal bar indicates \pm one standard deviation.

densidades relativamente altas en todas las zonas intermareales (tabla 1).

No se encontraron patelas por encima de los $2.5 \mathrm{~m}$ MLW, por lo tanto, la diferencia de densidad debido a la altura intermareal no afectó el diseño experimental. Los valores promedio de la longitud de abertura, altura y grosor de la concha se sintetizan en la tabla 2. El ANOVA indicó que la concha de $N$. biradiata fue más grande cuando se encontró en zonas de baja densidad de gasterópodos intermareales (longitud de abertura: $F_{1,163}=13.75, P<0.001$; altura: $\left.F_{1,163}=6.15, P<0.05\right)$, mientras que la altura intermareal no fue fuente de varianza para la longitud o altura de la concha (longitud de abertura: $F_{1,163}=$ $0.39, P>0.50$; altura: $F_{1,163}=0.21, P>0.50$ ). El grosor de la concha no cumplió la condición de homocedasticidad de varianza aún después de la transformación logarítmica (Bartlett, $X^{2}=2.66, P<0.001$ ). No se detectaron diferencias significativas en el grosor de la concha ni por efecto de la densidad (Kruskall-Wallis, $X^{2}=0.39, P>0.50$ ) ni de la altura intermareal (Kruskall-Wallis, $X^{2}=1.30, P>0.20$ ).

El análisis de regresión lineal entre la longitud de aberturaaltura y longitud de abertura-grosor de la concha se realizó en función a la densidad de gasterópodos intermareales. Todas las asociaciones fueron significativas (Ensenada de Utría: alturalongitud de abertura $F_{1,75}=14.52, P<0.001$ y grosor-longitud de abertura $F_{1,75}=14.96, P<0.001$; Isla Gorgona: alturalongitud de abertura $F_{1,87}=182.35, P<0.001$ y grosorlongitud $F_{1,87}=33.45, P<0.001$ ) (fig. 3 ). El ANCOVA sugiere que la asociación entre longitud de abertura-altura corresponde a una respuesta morfológica diferenciada por efecto de la densidad $\left(F_{1,162}=14.41, P<0.001\right)$, mientras que la asociación

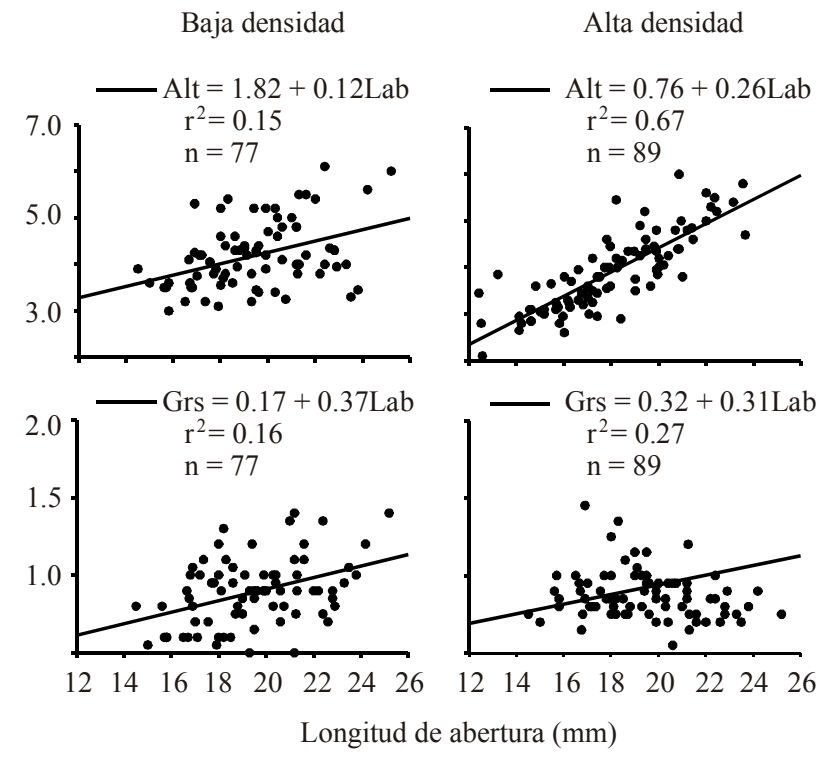

Figura 3. Regresiones lineales ajustadas de la longitud de la abertura vs altura y longitud de la abertura vs grosor de la concha de Notoacmea biradiata a baja y alta densidad de gasterópodos intermareales.

Figure 3. Fitted Linear regressions of shell length vs shell height and shell length vs thickness for Notoacmea biradiata at low and high density of intertidal gastropod.

height, being most abundant in the high intertidal zone $\left(F_{2,66}=8.94, P<0.001\right.$; Tukey-test: $\left.P<0.001\right)$. However, no significant variation in gastropod density was found between low and mid intertidal zones $\left(F_{2,66}=2.45, P>1.40\right)$. The intertidal gastropods observed during field samplings were mostly herbivorous species: limpets in the low and mid zones and littorines in the high zone. Though, predator gastropods were found in all intertidal zones and their densities were slightly high (table 1).

No limpets above $2.5 \mathrm{~m}$ MLW were found in any of the study sites, then the differences in density due to the intertidal height did not affect the statistical test. Basal shell-length, shell-height and shell-thickness mean values are synthesized in table 2. A two-way ANOVA test on basal shell-length and shell-height showed that the $N$. biradiata shell was bigger at low intertidal gastropod density (basal shell-length: $F_{1,163}=13.75, P<0.001$; shell-height: $F_{1,163}=6.15, P<0.05$ ). The intertidal shore height was not a significant source of variance for basal shell-length or shell-height (basal shell-length: $F_{1,163}=0.39, P>0.50$; shell-height: $F_{1,163}=0.21, P>0.50$ ). The homoscedasticity condition was not fulfilled by shellthickness even with logarithmic transformation (Bartlett, $\left.X^{2}=2.66, P<0.001\right)$. No significant differences in shell-thickness between densities (Kruskall-Wallis, $X^{2}=0.39, P>0.50$ ) or between intertidal shore heights (Kruskall-Wallis, $X^{2}=1.30$, $P>0.20$ ) were detected.

The least-square regressions of basal shell-length $v s$ shellheight and basal shell-length $v s$ shell-thickness were examined by density level. All associations were significant (Utría Cove: basal shell-length $v s$ shell-height $F_{1,75}=14.52, P<0.001$ and 
Tabla 1. Densidad de gasterópodos intermareales en la playa rocosa de la Ensenada de Utría e Isla Gorgona, en el Pacífico colombiano, agrupadas por zona intermareal. PR1 = Playa Blanca, PR2 = La Camaronera, PR3 = Playa Salomón, PR4 = Cocalito; $D=$ Densidad (no. ind $\left.\mathrm{m}^{-2}\right) ; S D=$ Desviación estandar de $D ; I C=$ Intervalo de confianza de $\mathrm{D} ; 10=$ Índice de ocurrencia $(\%)$.

Table 1. Intertidal gastropods density from Utría Cove and Gorgona Island rocky shores in the Colombian pacific coast clustered by intertidal zone. PR1 = Playa Blanca, PR2 = La Camaronera, PR3 = Playa Salomón, PR4 = Cocalito. $D=$ Density (no. ind $\mathrm{m}^{-2}$ ) $; \mathrm{SD}=\mathrm{Standard}$ deviation of $D ; I C=$ Confidence interval of $D: I O=$ Ocurrence index $(\%)$.

\begin{tabular}{|c|c|c|c|c|c|c|}
\hline Playa Rocosa & Zona Intermareal & Especie & $D$ & $S D$ & IC & $I O$ \\
\hline \multirow[t]{14}{*}{ PR1 - PR2 } & \multirow[t]{10}{*}{ Baja - Media } & Littorina aspera & 27.34 & 0.34 & 0.77 & 50 \\
\hline & & Fissurella virescens & 23.70 & 0.28 & 0.64 & 58 \\
\hline & & Nerita scabricosta & 16.66 & 0.16 & 0.37 & 54 \\
\hline & & Scurria stipulata & 12.24 & 0.15 & 0.34 & 63 \\
\hline & & Notoacmea biradiata & 2.08 & 0.04 & 0.09 & 21 \\
\hline & & Acanthina brevidentata & 1.30 & 0.05 & 0.10 & 8 \\
\hline & & Purpura pansa & 0.78 & 0.02 & 0.04 & 13 \\
\hline & & Tegula sp. & 0.78 & 0.02 & 0.05 & 8 \\
\hline & & Thais melones & 0.52 & 0.02 & 0.05 & 4 \\
\hline & & Mitrella elegans & 0.26 & 0.01 & 0.03 & 4 \\
\hline & \multirow[t]{4}{*}{ Alta } & Nerita scabricosta & 22.66 & 0.25 & 0.57 & 46 \\
\hline & & Littorina aspera & 17.19 & 0.28 & 0.64 & 33 \\
\hline & & Scurria stipulata & 5.73 & 0.12 & 0.27 & 25 \\
\hline & & Purpura pansa & 1.56 & 0.04 & 0.08 & 17 \\
\hline \multirow[t]{17}{*}{ PR3 - PR4 } & \multirow[t]{11}{*}{ Baja - Media } & Shiponaria maura & 6.25 & 0.10 & 0.23 & 33 \\
\hline & & Fissurella virescens & 5.57 & 0.07 & 0.15 & 42 \\
\hline & & Fissurella microtrema & 3.39 & 0.04 & 0.09 & 42 \\
\hline & & Acanthina brevidentata & 3.13 & 0.06 & 0.14 & 25 \\
\hline & & Siphonaria gigas & 1.82 & 0.04 & 0.09 & 17 \\
\hline & & Notoacmea biradiata & 1.82 & 0.03 & 0.08 & 21 \\
\hline & & Nerita funiculata & 1.30 & 0.04 & 0.08 & 13 \\
\hline & & Thais melones & 1.30 & 0.03 & 0.06 & 17 \\
\hline & & Nerita scabricosta & 0.78 & 0.02 & 0.06 & 8 \\
\hline & & Purpura pansa & 0.78 & 0.02 & 0.04 & 13 \\
\hline & & Scurria Mesoleuca & 0.52 & 0.02 & 0.03 & 8 \\
\hline & \multirow[t]{6}{*}{ Alta } & Nerita scabricosta & 14.32 & 0.19 & 0.43 & 38 \\
\hline & & Littorina aspera & 13.28 & 0.32 & 0.71 & 29 \\
\hline & & Nerita funiculata & 4.95 & 0.10 & 0.24 & 25 \\
\hline & & Littorina modesta & 3.91 & 0.08 & 0.18 & 25 \\
\hline & & Purpura pansa & 0.26 & 0.01 & 0.03 & 4 \\
\hline & & Thais melones & 0.26 & 0.01 & 0.03 & 4 \\
\hline
\end{tabular}


Tabla 2. Valor promedio de la longitud de abertura, altura y grosor de la concha de Notoacmea biradiata en dos localidades de la costa pacífica colombiana. $n=$ tamaño de muestra, $m=$ promedio, $D S=$ desviación estándar.

Table 2. Mean basal shell-lenght, shell-height and shell-thickness of Notoacmea biradiata from two localities in the Colombian pacific coast. $n=$ Sample size, $m=$ Mean, $D S=$ Standard deviation .

\begin{tabular}{|c|c|c|c|c|c|c|c|}
\hline Densidad & Zona Intermareal & Parámetro de la concha & $n$ & $m$ & $D S$ & Mínimo & Máximo \\
\hline \multirow[t]{3}{*}{ Baja } & Baja & Longitud de abertura & 42 & 19.05 & 2.56 & 15.60 & 25.20 \\
\hline & & Altura & 42 & 4.24 & 0.76 & 3.00 & 6.10 \\
\hline & & Grosor & 42 & 0.95 & 0.20 & 0.60 & 1.40 \\
\hline \multirow[t]{3}{*}{ Baja } & Media & Longitud de abertura & 35 & 18.88 & 1.98 & 14.50 & 22.60 \\
\hline & & Altura & 35 & 4.16 & 0.67 & 3.10 & 5.40 \\
\hline & & Grosor & 35 & 0.81 & 0.21 & 0.50 & 1.40 \\
\hline \multirow[t]{3}{*}{ Alta } & Baja & Longitud de abertura & 47 & 17.78 & 2.87 & 12.40 & 23.55 \\
\hline & & Altura & 47 & 3.82 & 0.88 & 2.10 & 5.80 \\
\hline & & Grosor & 47 & 0.85 & 0.13 & 0.65 & 1.20 \\
\hline \multirow[t]{3}{*}{ Alta } & Media & Longitud de abertura & 42 & 18.20 & 2.40 & 12.50 & 23.65 \\
\hline & & Altura & 42 & 3.99 & 0.78 & 2.80 & 6.00 \\
\hline & & Grosor & 42 & 0.91 & 0.18 & 0.55 & 1.45 \\
\hline
\end{tabular}

entre la longitud de abertura-grosor no fue estadísticamente diferente $\left(F_{1,162}=0.30, P>0.50\right)$.

En resumen, los resultados de esta investigación sugieren que: (a) la densidad de gasterópodos intermareales en las playas rocosas de la Ensenada de Utría y la Isla Gorgona son significativamente diferentes; (b) no hay diferencias significativas de densidad entre la zona intermareal baja y la zona intermareal media; (c) existe una estrecha relación entre la variabilidad del tamaño de la concha de $N$. biradiata con la densidad de gasterópodos, encontrando que la concha es más grande (mayor abertura y altura) cuando hay baja densidad; y (d) la altura intermareal no es un factor determinante de la variabilidad del tamaño de la concha de $N$. biradiata.

\section{Discusión}

La Isla Gorgona y la Ensenada de Utría no presentan diferencias ambientales significativas que permitan explicar la diferencia en la densidad de gasterópodos intermareales encontrada en este estudio. Sin embargo, dos factores no considerados en esta investigación podrían estar desempeñando un papel determinante para explicar estas diferencias en densidad: el impacto antrópico sobre la zona intermareal, y la intensidad de predación natural.

Las comunidades humanas residentes en la zona costera de la Ensenada de Utría utilizan frecuentemente diferentes especies de gasterópodos intermareales (e.g., patelas y neritas) como complemento a su dieta alimentaria (A. Giraldo, com. pers.); mientras que en la Isla Gorgona no hay asentamientos basal shell-length $v s$ shell-thickness $F_{1,75}=14.96, P<0.001$; Gorgona Island: basal shell-length $v s$ shell-height $F_{1,87}=182.35, P<0.001$ and basal shell-length $v s$ shellthickness $F_{1,87}=33.45, P<0.001$ ) (fig. 3). The ANCOVA test indicated that basal shell-length $v s$ shell-height association correspond to differentiated morphological responses to the density effect $\left(F_{1,162}=14.41, P<0.001\right)$, while basal shell-length $v s$ shell-thickness regression was not significantly different $\left(F_{1,162}=0.30, P>0.50\right)$.

In short, these results suggest that: (a) the intertidal gastropod density between Utría Cove and Gorgona Island rocky shores was significantly different; (b) there is not gastropod density difference between the mid and the low intertidal zones; (c) the shell-size variation of $N$. biradiata was associated with intertidal gastropod density, with bigger shells (greater basal shell-length and shell-height) being found in low intertidal gastropod density; and (d) shore height did not show a significant effect on the shell-size variation for $N$. biradiata.

\section{Discussion}

Gorgona Island and Utría Cove did not show important environmental differences that explain the intertidal gastropod density variation found in this study. However, two factors that were not considered in this research should have an important role in the explanation of this density variation: the human impact on intertidal gastropods, and natural predation.

Human communities, inhabiting in the coastal zone of Utría 
humanos permanentes y el acceso de personas es controlado por el Ministerio del Medio Ambiente de la República de Colombia. Por otra parte, la intensidad de la predación natural afecta directamente la densidad de gasterópodos en la zona intermareal tropical (Palmer, 1979; Garrity y Levings, 1981). En términos generales, los gasterópodos intermareales se encuentran expuestos a dos grupos principales de predadores, los peces y los crustáceos (Vermeij, 1978; Bertness y Cunningham, 1981). En la costa pacífica de Panamá, la mayoría de especies de invertebrados de la comunidad intermareal habitan en grietas, huecos y depresiones, en parte como resultado de la intensa predación por peces (Menge y Lubchenco, 1981), mientras que en la costa pacífica de Costa Rica la comunidad intermareal habita sobre superficies abiertas y los peces no parecen ser predadores importantes (Ortega, 1985). Considerando los resultados directos de esta investigación, la relación predador-presa en la comunidad intermareal de la costa pacífica colombiana podría estar influenciada por condiciones locales específicas. De acuerdo con las observaciones de campo, la ubicación de los gasterópodos en la zona intermareal rocosa de la Ensenada de Utría es similar a la reportada para Panamá por Menge y Lubchenco (1981); mientras que en la Isla Gorgona los gasterópodos se encontraron asociados principalmente a superficies abiertas, como lo reportó Ortega (1985) para Costa Rica.

La comunidad de gasterópodos intermareales en las áreas de estudio incluye principalmente patelas y litorinas (tabla 1). Los organismos que habitan en estas zonas parecen estar inmersos en un fuerte efecto denso-dependiente, derivado probablemente por la competencia alimenticia, la cual, puede afectar las tasas de mortalidad, crecimiento y reproducción de los gasterópodos (Paine, 1969). Por ejemplo, cuando las patelas se alimentan en el mismo rango intermareal, la competencia interespecífica e intraespecífica es fuerte, llegando incluso a alterar la estructura poblacional o la tasa de sobrevivencia (Underwood, 1979). Además, la zona intermareal tropical presenta condiciones extremas de temperatura durante la exposición directa al sol y el aire. La temperatura del sustrato puede exceder $35^{\circ} \mathrm{C}$ en días soleados y, en marea baja, el nivel letal de estrés térmico puede alcanzarse en superficies abiertas durante cualquier hora del día. Esto refleja la naturaleza transicional del hábitat intermareal en el incremento de la desecación y temperaturas extremas desde el nivel bajo al nivel alto de la costa. Por lo tanto, algunas especies de gasterópodos intermareales alteran la forma de su concha como una respuesta a la desecación (Branch, 1985). En este estudio la altura intermareal (e indirectamente la desecación), no afectó el tamaño de la concha de $N$. biradiata. Este resultado es contrario a lo reportado por Vermeij (1973), quien encontró una clara tendencia a la disminución del tamaño de la concha a medida que se incrementa la altura sobre la playa. Considerando que esta especie solo se encontró desde la zona intermareal media hacia abajo y que la altura intermareal no afecta el tamaño de su concha, es probable que el nivel de desecación que experimenta no alcanza el umbral crítico de tolerancia. Consecuentemente, no
Cove, use different intertidal gastropod species (e.g. patelas and nerites) in their daily diet (A. Giraldo, pers. comm.), while in Gorgona Island the access is restricted by the Environment Ministry of Colombia. On the other hand, the intensity of natural predation is another factor that affect gastropod density in the tropical intertidal zone (Palmer, 1979; Garrity and Levings, 1981). In the tropical zone, the intertidal gastropods are exposed to shell-breaking predation from two major feeding guilds: fishes and bottom crustaceans (Vermeij, 1978; Bertness and Cunningham, 1981). In the pacific coast of Panama, the invertebrate species of the intertidal community frequently occur in cracks, holes and depressions, partly as a result of intense fish predation (Menge and Lubchenco, 1981), while in the Pacific coast of Costa Rica, the intertidal community occurs on open surfaces, and fishes are not important predators (Ortega, 1985). Considering the direct results of the study of $N$. biradiata, the predator-prey relationship in the intertidal rocky shore of the Colombian pacific coast should be influenced by specific local conditions. Field observations indicated that intertidal gastropods distribution over the rocky shores of Utría Cove was similar to the Pacific coast of Panama (Menge and Lubchenco, 1981), while the spatial distribution of intertidal gastropods over the rocky shores of Gorgona Island were associated with open surfaces, similar to what has been observed in the Pacific coast of Costa Rica (Ortega, 1985).

The gastropod intertidal community in the areas studied includes mainly herbivorous limpets and littorines (table 1). The individuals that inhabit this zone show a strong densitydependent effect attributable to competition for food. This has a considerably effect on gastropod mortality, growth rates and reproduction (Paine, 1969); e.g., when limpets feed on microalgae in the same intertidal range, the interspecific and intraspecific competition for food is so stromg, that it will alter the community structure or the survival rates (Underwood, 1979). Furthermore, the tropical intertidal zone experiences continuous harsh physical conditions during daytime exposures to sun and air. Substratum temperatures can exceed $35^{\circ} \mathrm{C}$ in sunny weather, and lethal stress levels can be reached on open surfaces at any daytime in a clear day. This reflects the transitional nature of the intertidal habitat, increasing the rigor of desiccation and extreme temperatures from low to high shore heights. Therefore, many intertidal gastropod species change the shape of their shell in response to desiccation (Branch, 1985). In this study, the shell-size of $N$. biradiata was not affected by the intertidal shore height. The absence of an intertidal height effect on the shell-size was disagreeing with Vermeij's (1973) study, which reported a negative relationship between shell-size and intertidal height. Since $N$. biradiata was only found from mid to low intertidal zones and the intertidal height did not affect the shell sizes, it is probable that the upper threshold of desiccation was not reached for this specie in the study zone. Thus, the shell of $N$. biradiata does not express a morphological response to the effect of this modifying factor.

Strong evidence that gastropod density is the most likely 
expresa una respuesta morfológica al efecto de este factor modificador.

Este estudio presenta considerable evidencia que sugiere que la densidad de gasterópodos es uno de los factores que afecta directamente la variación del tamaño de la concha de $N$. biradiata en la costa pacífica colombiana, siendo más grande y más alta en zonas de baja densidad de gasterópodos. Es de esperar que, cuando la densidad de gasterópodos es baja, el alimento disponible sea mayor $\mathrm{y}$, por lo tanto, es probable que la tasa de crecimiento se incremente, dando como resultado una concha grande y alta (Branch, 1975b). Sin embargo, las influencias de otros factores físicos o biológicos deberían ser tenidas en cuenta. Recientemente, Gómez et al. (2001) encontraron que el grosor de la concha de $N$. biradiata responde a la intensidad del oleaje, siendo las conchas más gruesas en zonas de alta energía dinámica. Trabajos ecológicos futuros en el intermareal rocoso del Pacífico colombiano, podrán evaluar el efecto de otros factores biológicos y ambientales sobre las características morfológicas de los gasterópodos intermareales. Implementar estos nuevos trabajos, en escalas espaciotemporales pertinentes, es crítico para avanzar en el entendimiento de las respuestas biológicas de los gasterópodos al efecto de las interacciones físico-biológicas del ambiente en el intermareal rocoso tropical (Chapman, 2000; Underwood, 2000).

\section{Agradecimientos}

Este estudio fue parcialmente financiado por el Departamento de Biología de la Universidad del Valle. El Ministerio del Medio Ambiente permitió el acceso a los Parques Naturales Isla Gorgona y la Ensenada de Utría para el estudio de campo. El Departamento Alemán de Intercambio Académico (DAAD) financió a A.G. y E.R. durante sus estudios de postgrado en la Universidad de Concepción-Chile.

\section{Referencias}

Anónimo (1983). Atlas Regional del Pacífico. Instituto Geográfico Agustín Codazzi, Bogotá, Colombia, 26 pp.

Bertness, M.D., Garrity, S.D. and Levings, S.C. (1981). Pressure and gastropods foraging: a tropical-temperature comparison. Evolution, 35: 995-1007.

Bertness, M.D. and Cunningham, C. (1981). Crab shell crushing predation and gastropod architecture. J. Exp. Mar. Biol. Ecol., 50: 213-230.

Black, R. (1979). Competition between intertidal limpets: an intrusive niche on a steep resource gradient. J. Anim. Ecol., 48: 401-411.

Blackford, S. and Cook, C. (1978). Tidal amplitude and activity in the pulmonated limpets Shiponaria normalis Gould and S. Alternata Say. J. Exp. Mar. Biol. Ecol., 35: 119-136.

Butler, A.J. (1979). Relationships between height on the shore and size distributions of Thais sp. Gastropoda: Muricidae. J. Exp. Mar. Biol. Ecol., 41: 163-194.

Branch, G.M. (1975a). Ecology of Patella species from Cape Peninsula, South Africa. IV. Desiccation. Mar. Biol., 32: 179-188.

Branch, G.M. (1975b). Intraspecific competition in Patella cochlear agent of shell-size variation in $N$. biradiata of the Pacific coast of Colombia was showed in this study. The shell of this limpet was bigger and higher at low intertidal gastropod densities. It is expected that an appropriate food quantity should be available for the limpets' growth when the intertidal gastropod density is low. Then, the growth rate should be high resulting in a big and tall shell (Branch, 1975b). However, the influence of other physical and biological factors should be tested too. Recently, Gómez et al., (2001) reported the variation of $N$. biradiata shell-thickness due to wave intensity, being the shell thicker in exposed rocky shores. Future ecological research in the rocky intertidal zone of the Pacific coast of Colombia should evaluate the effects of other biological and environmental factors on the shell characteristics of intertidal gastropods. The implementation of new projects in relevant time-space scales is critical to advance in the understanding of the gastropod responses to the effects of physical-biological interactions in the tropical intertidal rocky shore (Chapman, 2000; Underwood, 2000).

\section{Acknowledgements}

This study was partially funded by the Investigation Division of the University of Valle. The Colombian Environmental Ministry granted the access to the Gorgona Island and Utría Cove national parks. The German Department of Academic Exchange (DAAD) provided support for the authors during their postgraduate studies at University of Concepción-Chile.

Englsh translation by the authors.

Born. J. Anim. Ecol., 44: 263-282.

Branch, G.M. (1985). Limpets: evolution and adaptation. The Mollusca, 10: 187-220.

Branch, G.M. and Cherry, M.I. (1985). Activity rhythms of the pulmonate limpet Shiponaria capensis Q. and G. as an adaptation to osmotic stress, predation and wave action. J. Exp. Mar. Biol. Ecol., 87: 153-168.

Brown, M.K. and Quinn, J.F. (1988). The effect of wave action on growth in three species of intertidal gastropods. Oecology, 75: 420-425.

Chapman, M.G. (2000). Poor design of behavioural experiments gets poor results: examples from intertidal habitats. J. Exp. Mar. Biol. Ecol, 250: 77-95.

De Wolf, H., Brito, C., Van Dongen, S. and Backeljau, T. (1999). Did heavy storm affect the aperture area of wave-exposed and sheltered Littorina striata? J. Mar. Biol. Ass. U.K, 79: 1129-1130.

Denny, M.W. (1994). Roles of hydrodinamics in the study of life on wave-swept shores. In: P. Wainwright and R. Stephen (eds). Ecological Morphology, Integrative Organismal Biology. Univ. of Chicago Press, pp. 169-204.

Edbling, F.J., Sloang, J.F. and Davies, H.M. (1962). The ecology of lough line. XII. The distribution and characteristics of Patella species. J. Anim. Ecol., 31: 457-470.

Forsbergh, E.D. (1969). On the climatology, oceanography and 
fisheries of the Panama Bight. Bull. I-ATTC, 14: 49-365.

Frank, P.W. (1965). The biodemography of an intertidal snail population. Ecology, 46: 831-844.

Garrity, S.D. and Levings, S.C. (1981). A predator-prey interaction between two physically and biologically constrained tropical rocky shore gastropods: direct, indirect and community effects. Ecol. Monogr, 51: 267-286.

Garrity, S.D. and Levings, S.C. (1983). Homing scars as a defense against predators in the pulmonated limped Siphonaria gigas Gastropoda. Mar. Biol, 72: 319-324.

Giraldo, A. y Gómez, C. (1999). Shell variation of Shiponaria gigas Sowerby, 1825, in response to the effect of wave intensity. Ciencias Marinas, 25: 213-224.

Gómez, C., Giraldo, A. y Rodríguez, E. (2001). Efecto de la intensidad del oleaje sobre la morfometría de Notoacmea biradiata (Archaeogastropoda: Acmaeidae) en el Pacífico colombiano. En: Resumen Extendido. IX Congreso Latinoamericano sobre Ciencias del Mar. San Andrés Isla, Colombia. 83 pp.

Heller, J. (1976). The effects of exposure and predation on the shell of two British winkles. J. Zool., 179: 201-213.

Hobday, A. (1995). Body-size variation exhibited by an intertidal limpet: influence of wave exposure, tidal height and migratory behavior. J. Exp. Mar. Biol. Ecol., 189: 29-45.

Keen, A.M. (1971). Seashells of tropical west america. 2nd ed. Stanford Univ. Press, Stanford, California, 1064 pp.

Levinton, J.S. (1984). Marine Ecology. Prentice Hall, Inc. New Jersey. $526 \mathrm{pp}$.

Lewis, R.J. (1964). The Ecology of Rocky Shore. London. English University Press. 323 pp.

Lowell, R.B. (1984). Desiccation of intertidal limpets: effects of shell size, fit to substratum, and shape. J. Exp. Mar. Biol. Ecol, 77; 197-207.

Menge, B.A. and Lubchenco, J. (1981). Community organization in temperate and tropical rocky intertidal habitats: prey refuges in relation to consumer pressure gradients. Ecol. Monogr., 51: 429450.

Nybakken, J.W. (1997). Marine Biology: An Ecological Approach. 4th ed. Addison - Wesley, $481 \mathrm{pp}$.

Ortega, S. (1985). Competitive interactions among tropical intertidal limpets. J. Exp. Mar. Biol. Ecol., 90: 11-25

Pabon, J.D., Rojas, P.E., Montealegre, J.E., Kim, R., Ceballos, J.L., Martinez, N. y Nanez, E. (1998). El Océano. En: P. Leyva (ed.), El Medio Ambiente en Colombia. Instituto de Hidrología, Meteorología y Estudios Ambientales, IDEAM. Santa Fé de Bogotá, pp. 149-169.

Paine, R.T. (1969). The Pisaster-Tegula interacction: prey patches, predator food preferences and intetidal community structure. Ecology, 50: 950-962.

Palmer, A.R. (1979). Fish predation and the evolution of gastropod shell sculpture: experimental and geographic evidence. Evolution, 33: 697-713.

Rodríguez-Rubio, E. (1997). Cartografía de unidades vegetales y geomorfología costera mediante la aplicación de sensores remotos en Isla Soldado, Pacífico colombiano. Tesis de grado, Universidad del Valle, Cali, Colombia, 78 pp.

Spencer, P. (1969). Physiological ecology of Patella. III. Desiccation effects. J. Mar. Biol. Ass. UK, 49: 291-304.

Tchantsev, V. y Cabrera, E. (1999). Algunos aspectos de investigación de la formación del régimen oceanográfico en el Pacífico colombiano. Boletín Científico CCCP, 7: 7-19.

Underwood, A.J. (1979). The ecology of intertidal gastropods. Adv. Mar. Biol., 16: 111-210.

Underwood, A.J. (2000). Experimental ecology of rocky intertidal habitats: what are we learning? J. Exp. Mar. Biol. Ecol., 250: 5176.

Vermeij, G.J. (1972). Intraespecific shore-level size gradients in intertidal molluscs. Ecology, 53: 693-700.

Vermeij, G.J. (1973). Morphological patterns in high-intertidal gastropods: adaptive strategies and their limitations. Mar. Biol., 20: 319-346.

Vermeij, G.J. (1978). Biogeography and Adaptation, Patterns of Marine Life. Harvard Univ. Press. Cambridge, Massachusetts. 332 pp.

Wolcott, T.G. (1973). Physiological ecology and intertidal zonation in limpets Acmaea: a critical look of limiting factors. Biol. Bull, 145: 389-422.

Zar, J.H. (1999). Biostatistical Analysis. 4th ed. Prentice Hall. London. 929 pp. 